\title{
Burnout, inteligencia emocional y rendimiento académico: un estudio en alumnado de medicina
}

Javier Casanova Diez, Sara Benedicto Álvaro, Fátima Luna Delgado y Cristina Maldonado Conchillo. Universidad de Granada

\author{
Recepción: 20 de mayo de 2016 | Revisión: 21 de mayo de 2016 | Aceptado: 30 de mayo de 2016 \\ Correspondencia: Javiercd94@correo.ugr.es \\ Citar: Casanova, J., Benedicto, S., Luna, F. y Maldonado, C. (2016). Burnout, inteligencia emocional y rendimiento académico: un \\ estudio en alumnado de medicina. ReiDoCrea, 5(2), 1-6.
}

\begin{abstract}
Resumen: El objetivo de la presente investigación es describir las relaciones existentes entre burnout, inteligencia emocional y rendimiento académico. Para ello se utilizó una muestra de 50 estudiantes de medicina de la universidad de Granada. Para evaluar el burnout y la inteligencia emocional se utilizaron los cuestionarios EUBE y TMMS-24 respectivamente, para evaluar el rendimiento académico se preguntó por la nota del expediente. Los resultados evidencian una relación positiva entre la subescala atención a las emociones y burnout en ambos sexos, y una relación positiva entre las subescalas claridad emocional y reparación de las emociones en hombres.
\end{abstract}

Palabras clave: Burnout | Inteligencia Emocional

Burnout, Emotional Intelligence and Academic Achievement: A Study on Medicine Students

Abstract: The purpose of this study is to describe the existing links between burnout, emotional intelligence and academic achievement. To that end, a sample of 50 medical students of the University of Granada was used. In order to assess burnout and emotional intelligence, EUBE and TMMS-24 questionnaires were used; To evaluate academic achievement, the grades of the academic records were requested. The results show, a positive relation between the subscale attention to emotions and burnout in both sexes, as well as a positive relation between the subscales of emotional clarity and emotional reparation in men.

Key words: Burnout | Emotional Intelligence

\section{Introducción}

En la actualidad, la experiencia de burnout goza de una amplia aceptación en el ámbito académico, hecho contrastado mediante la adaptación de instrumentos previos, y la elaboración de instrumentos propios (Barraza, 2009, 2011; Salanova, Bresó y Schaufeli, 2005). El síndrome está especialmente relacionado con las ciencias sanitarias, como por ejemplo enfermería y medicina (Caballero, Abello y Palacios, 2007). Además, existe evidencia de mayor ideación suicida en estudiantes de medicina con burnout en comparación con estudiantes de otros grados (Picasso-Pozo et al., 2012).

El síndrome de Burnout se abordó por primera vez en 1961 con la publicación de $A$ Burnout Case de Graham Greens (Borda, Navarro, Aun, Berdejo, Racedo y Ruiz, 2007). Sin embargo, el término Burnout se comenzó a utilizar en 1977, tras la exposición de Maslach ante una convención de la American Psychological Association, en la que conceptualizó el síndrome como el desgaste profesional de aquellas personas que trabajan en el sector servicios, especialmente sanitario y educativo (Martínez, 2010). De este modo, el síndrome es la respuesta al estrés crónico típico de la cotidianidad del trabajo, en situaciones de presión, conflicto y poco reconocimiento (Carlotto, Gonçaves y Brazil, 2005).

Según Barraza (2011), el desarrollo teórico-conceptual del burnout se divide en dos enfoques conceptuales diferentes: el primero se origina con el desarrollo y la difusión del trabajo de Maslach y Jackson (1981) que permitió conceptualizar el burnout como 
un constructo tridimensional. El segundo se inicia con el trabajo de Pines et al (1981) cuyo desarrollo aportó un enfoque unidimensional mediante el agotamiento emocional.

El enfoque multidimensional conceptualiza el burnout mediante sus tres componentes principales, agotamiento emocional, despersonalización y, autoeficacia (Cooper, 2002). Esta concepción del burnout se respalda en el posterior análisis de las propiedades psicométricas del Maslach Burnout Inventory (MBI) (Maslach, 1981). Por su parte, Pines, Aronson y Kafry (1981) definen el síndrome como un agotamiento físico, emocional y mental causado por la implicación personal durante largos periodos de tiempo en aquellas situaciones que le afecten emocionalmente (Moreno, 2007).

La conceptualización que se adopta en la presente investigación se adhiere al enfoque unidimensional; esta forma de conceptualizarlo recibió un fuerte impulso con el trabajo de Shirom, quién llevo a cabo una revisión de todos los estudios de validación del MBI, permitiéndole observar, que de las tres dimensiones, es el agotamiento emocional la que más correlaciona con las variables relacionadas con el trabajo (Rosales y Rosales, 2013). Atendiendo al enfoque seleccionado, y a que el presente estudio se desarrolla en estudiantes universitarios se hará uso de la escala unidimensional de burnout estudiantil (EUBE) (Barraza, 2011).

Según Extremera y Durán (2006) "en el ámbito educativo u organizacional, la inteligencia emocional (IE) desempeñaría un papel importante como recurso individual que permite estudiar la relación entre los procesos de estrés y burnout". La clasificación conceptual más aceptada de la IE distingue entre modelos mixtos y modelos de habilidad fundamentados en el procesamiento de la información. Por un lado, el modelo mixto concibe la IE como un conjunto de rasgos de la personalidad, capacidades socio-emocionales, y aspectos motivacionales y cognitivos, los más conocidos son el modelo de Goleman y Bar-On. Por otro lado, el modelo de habilidad define la IE como la habilidad que procesa la información relevante de las emociones, y que es independiente a los rasgos de la personalidad. Además, es considerado como el modelo con mayor fundamento empírico. Por ello, en la realización de este estudio se hará uso de la definición de IE acuñada por Mayer y Salovey (1997) (Fernández y Extremera, 2005), así como de la adaptación al castellano de sus instrumentos.

Por otra parte, numerosas investigaciones exhiben una relación positiva entre la IE y el rendimiento académico. Autores como Extremera y Fernández-Berrocal (2004), Montero, Villalobos y Valverde (2007), Pérez y Castejón (2006) y Schutte et al (1998) encuentran correlaciones empíricamente significativas entre inteligencia emocional y rendimiento académico en estudiantes universitarios. Contrariamente, autores como Núñez (en Hernández, 2005) niegan la capacidad predictiva de la IE sobre el rendimiento académico.

Todo lo expuesto anteriormente evidencia que el burnout constituye un problema en el plano académico, pudiendo afectar al desarrollo de estudiantes universitarios. Los costes y repercusiones que conlleva a nivel individual, social y organizacional (Martínez, 2010), así como el limitado número de investigaciones en el ámbito académico justifican la necesidad de llevar a cabo un estudio, en el que además se tengan en cuenta la inteligencia emocional y el rendimiento académico. De este modo, el presente estudio busca describir las relaciones existentes entre el burnout, la IE, y el rendimiento académico. Así, el mismo plantea que mayores niveles de burnout estarán relacionados con niveles más bajos de IE y con un bajo rendimiento, mientras que la IE correlacionará positivamente con el rendimiento académico. 


\section{Método}

El estudio se llevó a cabo con una muestra compuesta por 50 estudiantes de medicina en la universidad de Granada. Del total, el $52 \%$ corresponde a mujeres, mientras que el $48 \%$ restante a hombres, todos con edades comprendidas entre los 18 y 24 años. En relación al nivel de formación, el $28 \%$ pertenecen a segundo, el $44 \%$ a tercero, el $12 \%$ a cuarto y, el $16 \%$ a quinto, todos ellos del turno de mañana.

\section{Instrumentos}

En la evaluación de la IE, se ha hecho uso del Traid Meta-Mood Scale (TMMS; Salovey et al, 1995) en su versión española TMMS-24 (Fernández-Berrocal, Extremera y Ramos, 2004). Es una escala tipo Likert con 5 opciones de respuesta que aporta información de las creencias personales de atención a las emociones y de la capacidad de regulación de éstas. Está compuesta por tres dimensiones, formadas por 8 ítems cada una: Atención emocional, claridad emocional y reparación de las emociones. La fiabilidad de este instrumento se encuentra por encima de .80 en cada dimensión (Atención $\alpha$ de Cronbach $=.84$; Claridad $\alpha$ de Cronbach $=.91$; y Reparación a de Cronbach $=.84)$. En la evaluación del burnout, se ha utilizado la Escala Unidimensional de Burnout Estudiantil (EUBE; Barraza, 2009, 2011). Es un inventario tipo Likert con cuatro opciones de respuesta. Está formado por 15 ítems que miden el agotamiento emocional. Este instrumento reporta una fiabilidad por mitades de $.90 \mathrm{y}$ un a de Cronbach de .91, similares a las de Barraza (2011). En la evaluación del rendimiento académico, se preguntó por la nota del expediente académico obtenida hasta el momento.

\section{Procedimiento}

Los datos se recogieron durante el primer cuatrimestre del curso académico 2015/2016. Dos de los autores de este trabajo repartieron los cuestionarios a cumplimentar junto con el consentimiento informado en la cafetería de la facultad, concediendo el tiempo oportuno para su realización.

\section{Diseño y análisis de datos}

El presente estudio ha utilizado un diseño correlacional básico, empleando el coeficiente de correlación lineal de Pearson. Asimismo, se ha llevado a cabo una prueba $\mathrm{T}$ de Student para muestras independientes con el fin de establecer posibles diferencias de género.

\section{Resultados}

Los análisis oportunos se han llevado a cabo mediante el paquete estadístico SPSS 19.0 para Windows. Se han realizado análisis descriptivos, correlacionales y comparativos a fin de contrastar las hipótesis planteadas inicialmente.

\begin{tabular}{|cccccc|}
\hline \multicolumn{7}{c|}{ Tabla 1. Estadísticos descriptivos } \\
\hline NE & 50 & Mínimo & Máximo & Media & Desv. típ \\
\hline AE & 50 & 5.80 & 9.17 & 7.42 & .75 \\
CE & 10.00 & 40.00 & 26.12 & 5.55 \\
RE & 50 & 10.00 & 40.00 & 27.74 & 6.17 \\
EUBE & 50 & 16.00 & 39.00 & 28.22 & 6.00 \\
"Nota: EUBE: Escala unidimensional de burnout estudiantil; AE: Atención emocional; CE: Cansancio emocional; RE: Recuperación de las \\
emociones; NE: Nota expediente.
\end{tabular}


De acuerdo al baremo, las puntuaciones obtenidas en la tabla 1 reflejan que la muestra de estudiantes de medicina presenta niveles moderados de burnout $(M=31.58$; $D T=8.09)$. Por su parte, la inteligencia emocional se divide en tres dimensiones, cada una de las cuales atiende a un baremo específico, situándose la muestra en unos niveles adecuados en todas las dimensiones ( $A E$ : $M=26.12$; $D T=$ 5.55, CE: $M=27.74$; $\mathrm{DT}=6.17$, RE: $\mathrm{M}=28.22$; $\mathrm{DT}=6.00$ ). Asimismo, la puntuación promedia en rendimiento académico es 7.42 con una desviación típica de .76.

En la tabla 2 se exponen los resultados obtenidos tras analizar las relaciones existentes entre las variables burnout, IE, y rendimiento académico mediante el método de correlación lineal de Pearson. De este modo, y con el objetivo de establecer diferencias de género en las correlaciones, se segmentó la muestra atendiendo a la variable "sexo".

\begin{tabular}{|c|c|c|c|c|c|}
\hline \multicolumn{6}{|c|}{ Tabla 2. Correlación de las variables de Inteligencia Emocional, Burnout (EUBE) y Rendimiento Académico } \\
\hline Mujeres / Hombres & $\mathrm{NE}$ & $\mathrm{AE}$ & $\mathrm{CE}$ & $\mathrm{RE}$ & EUBE \\
\hline $\mathrm{NE}$ & - & $-0,093$ & 0,226 & 0,148 & 0,234 \\
\hline $\mathrm{AE}$ & 0,048 & - & 0,122 & $-0,336$ & $0,415^{*}$ \\
\hline $\mathrm{CE}$ & 0,340 & 0,019 & - & $0,594^{* *}$ & 0,006 \\
\hline $\mathrm{RE}$ & 0,149 & $-0,061$ & 0,212 & - & 0,014 \\
\hline EUBE & $-0,070$ & $0,420^{*}$ & $-0,271$ & $-0,147$ & - \\
\hline \multirow{7}{*}{ La correlación es significante al nivel 0,05 (bilateral) } \\
* La correlación es significativa al nivel 0,01 (bilateral)
\end{tabular}

Tanto en mujeres como en hombres, se observa una correlación significativamente positiva entre la subescala $A E$ de inteligencia emocional y la puntuación total del EUBE $(r=.42, p<.05 ; r=.42, p<.05)$. Además, en hombres también correlacionan positivamente la subescala CE y la subescala RE de inteligencia emocional $(r=.59 ; p$ .01) En cuanto al resto de variables, no se han encontrado relaciones significativas.

Con el objetivo de analizar las diferencias de género en la muestra se llevó a cabo una prueba $T$ de Student para muestras independientes. Esta prueba reflejó que no existen diferencias de género en ninguna de las variables analizadas (EUBE: $t(48)=.588, p=$ .559 ; AE: $t(48)=-.461, p=.647$; CE: $t(48)=-1.07, p=.291$; RE: $t(48)=-.80, p=.936$; NE: $t(48)=.166, p=.869)$. A continuación se exponen en la figura 1 dichas diferencias.

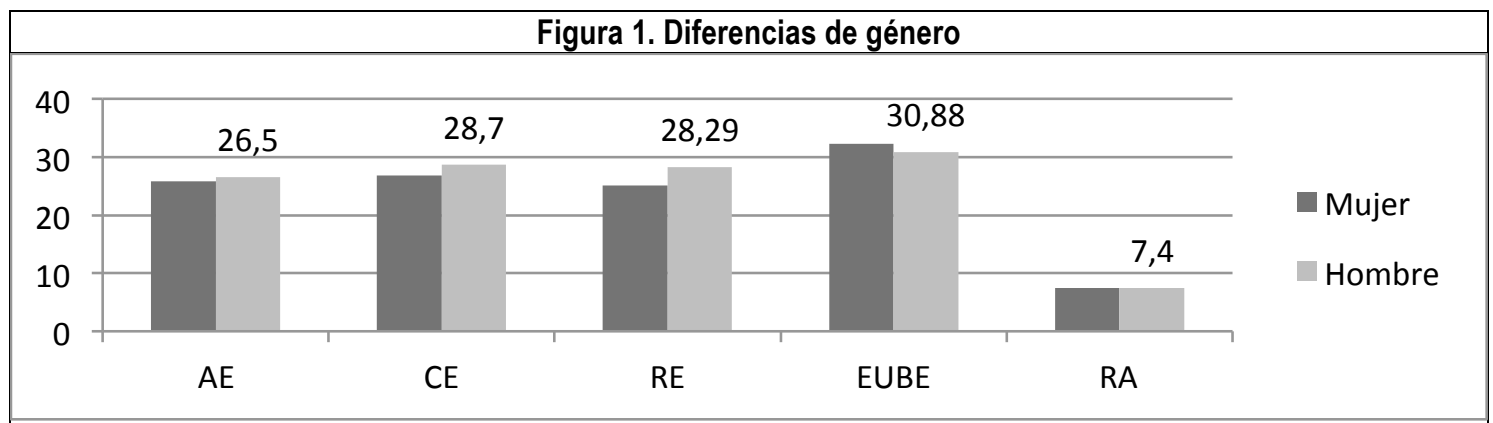

*Nota: EUBE: Escala unidimensional de burnout estudiantil; AE: Atención emocional; CE: Cansancio emocional; RE: Recuperación de las emociones; NE: Nota expediente; Eje Y: Medias; Eje X: Variables

\section{Discusión}

Diversos autores, como por ejemplo Extremera y Durán (2006) concluyen que la inteligencia emocional correlaciona negativamente con burnout, constituyendo así un valioso recurso personal. Sin embargo, tras realizar el análisis correlacional se han 
obtenido resultados contradictorios, debido a la carencia de resultados significativos en relación con el burnout en dos de las tres subescalas de IE (CE, RE), aunque si se observan tendencias estadísticas en la dirección planteada inicialmente, asimismo la dimensión atención a las emociones (AE) se ha relacionado positivamente con este síndrome. Un estudio en profesores de Extremera, Fernández-Berrocal y Duran (2003) planteó como hipótesis que la $A E$ se relacionaría positivamente con algunos componentes del burnout, sin embargo no obtuvieron resultados significativos. En este sentido, los resultados, podrían explicarse mediante el enfoque suscrito por la presente investigación, y a los instrumentos utilizados en ella. Debido a la inexistencia de publicaciones que relacionen burnout desde una perspectiva unidimensional con IE, la bibliografía consultada trata dicha relación desde el enfoque multidimensional de Masclach y Jackson (1981). Por su parte, en hombres, la dimensión CE correlaciona positivamente con RE, relación respaldada por Ortiz-Acosta y Beltrán-Jiménez (2011). No obstante, esta relación no se encuentra en mujeres, probablemente debido al reducido carácter de la muestra.

En cuanto al rendimiento académico, no se han observado relaciones significativas con ninguna variable. Estos datos se respaldan tanto en burnout como en IE, donde la variedad de publicaciones genera resultados contrapuestos atendiendo a las variables seleccionadas. Según Caballero, Cecilia y Abello (2007) el rendimiento académico no correlaciona con el componente agotamiento, mientras que correlacionó negativamente con cinismo, y positivamente con autoeficacia percibida. De nuevo, la inexistencia de estudios previos que hagan uso del enfoque unidimensional, dificulta la comprensión de los resultados obtenidos. Por su parte, numerosos estudios concluyen con la ausencia de relación entre IE y rendimiento académico (Núñez citado en Hernández, 2005). Además, los resultados obtenidos podrían ser fruto de la manipulación de los datos referidos por la muestra en esta variable.

Llegado a este punto, cabe mencionar los resultados obtenidos en la prueba $T$ de Student, según los cuales no se observan diferencias de género para ninguna variable. En lo que respecta al burnout estudios como el de Extremera y Durán (2006) apoyan estos resultados, sin embargo si se establecen diferencias de género en $A E$ y en RE, a favor de mujeres y hombres respectivamente. Así mismo, en lo que respecta al rendimiento existe cierta tendencia que sitúa las mujeres por encima de los hombres (Garbanzo, 2007). Al igual que las diferencias en correlación, los pobres resultados obtenidos pueden deberse al reducido carácter de la muestra.

En conclusión, en la presente investigación, los resultados obtenidos no respaldan las hipótesis planteadas inicialmente y contrarían la literatura abarcada en el desarrollo teórico-conceptual de las variables a relacionar. Por ello, es necesario desde el ámbito de la investigación realizar estudios que tengan en cuenta el enfoque unidimensional, con el resto de variables, con el fin de contrastar los resultados obtenidos en esta investigación. Asimismo, la presente finaliza con la idea de llevar a cabo un segundo estudio, ampliando la muestra.

\section{Referencias}

Barraza, A. (2009). Estrés académico y burnout estudiantil. Análisis de su relación en alumnos de licenciatura. Psicogente, 12(22), 272-283.

Barraza, A. (2011). Validación psicométrica de la escala unidimensional del burnout estudiantil. Revista Internacional de Psicología y Educación, 13(2), 51-74.

Borda, M., Navarro, E., Aun, E., Berdejo, H., Racedo, K., \& Ruiz, J. (2007). Burnout Syndrome in intership students at Hospital Universidad del Norte. Revista Salud Uninorte, 23(1), 43-51. 
Caballero, D., Cecilia, C., Abello, L.L. y Palacio, S. (2007). Relación del burnout y el rendimiento académico con la satisfacción frente a los estudios en estudiantes universitarios. Avances en Psicología Latinoamericana, 25(2), 98-111.

Carlotto, MS., Gonçalvez, S. y Brazil, AM. (2005). Predictores del síndrome de burnout en estudiantes de un curso técnico de enfermería. Perspectivas en Psicología, 1(2), 195-205.

Cooper, C. L. (2002). Theories of organizational stress. Estados Unidos: Oxford University Press.

Extremera, N. y Durán, A. (2006). Inteligencia emocional y su relación con los niveles de burnout, engagement y estrés en estudiantes universitarios. Revista de Educación, 372, 239-256.

Extremera, N., Fernández-Berrocal, P., Durán, A. (2003). Inteligencia emocional y burnout en profesores. Encuentros en psicología social, 1, 260-265.

Extremera, N., y Fernández-Berrocal, P. (2004). El papel de la inteligencia emocional en el alumnado: Evidencias empíricas. Revista Electrónica de Investigación Educativa, 6 (2), 1-17.

Fernández, P. y Extremera, N. (2005). La inteligencia emocional y la educación de las emociones desde el modelo de Mayer y Salovey. Revista Interuniversitaria de Formación del Profesorado, 19, (3), 63-93.

Garbanzo, G. M. (2007). Factores asociados al rendimiento académico en estudiantes universitarios, una reflexión desde la calidad de la educación superior pública. Revista educación, 31(1), 43-63.

Hernández, P. (2005). ¿Puede la inteligencia emocional predecir el rendimiento? Potencial predictor de los moldes mentales. Revista interuniversitaria de formación del profesorado, 19 (3), 45-62.

López, C. (2011). Relación de la inteligencia emocional con el desempeño en los estudiantes de enfermería. Tesis Doctoral. Cádiz: Universidad de Cádiz.

Martínez, A. (2010). El síndrome de burnout. Evolución conceptual y estado actual de la cuestión. Vivat Academia, 112, $1-40$.

Maslach, C., \& Jackson, S. E. (1981). The measurement of experienced burnout. Journal of occupacional behaviour, 2 , 99-113.

Moreno, A. (2007). Burnout asistencial: Identificación, prevención y mediación en factores precipitantes. Revista Electrónica de Psicología Iztacala, 10 (1), 63-79.

Ortiz-Acosta, R. y Beltran-Jimenez, B.E. (2011). Inteligencia emocional percibida y desgaste laboral en médicos internos de pregrado. Educación médica, 14(1), 49-55.

Pérez, N. y Castejón, JL. (2006). Relaciones entre la inteligencia emocional y el cociente intelectual con el rendimiento académico en estudiantes universitarios. Revista Electrónica de Motivación y Emoción, 9(22), 1-27.

Picasso-Pozo, M., Huillca-Castillo, N., Ávalos-Marquez, J., Omori-Mitumori, E., Gallardo-Schultz, A. y Salas-Chavez, M. (2012). Síndrome de Burnout en estudiantes de Odontología de una Universidad Peruana. Kiru, 9(1), 51-58.

Rojas, M. Villalobos, J. y Valverde, A. (2007). Factores institucionales, pedagógicos, psicosociales y sociodemográficos asociados al rendimiento académico en la universidad de costa rica: Un análisis multinivel. RELIEVE, 13(2), 215-234.

Rosales, Y. y Rosales, FR. (2013). Burnout estudiantil universitario. Conceptualización y estudio. Salud Mental, 36 (4), 337-345.

Salanova, M., Breso, E. y Schaufeli, W. B. (2005). Hacia un modelo espiral de las creencias de eficacia en el estudio del burnout y del engagedment. Ansiedad y estres, 11(2-3), 215-235.

Schutte, N. S., Malouff, J. M., Hall, L. E., Haggerty, D. J., Cooper, J. T., Golden, J., \& Dornheim, L. (1998). Development and validation of measure of emotional intelligence. Personality and Individual Differences, 25, 167-177. 